\title{
Implications of Bacterial Biofilms in Chronic Rhinosinusitis
}

\author{
Edwin Tamashiro $^{1,2}$, Marcelo B. Antunes ${ }^{2}$, James N. Palmer ${ }^{2}$, Noam A. Cohen ${ }^{2}$ and Wilma T. Anselmo-Lima ${ }^{1}$ \\ ${ }^{1}$ Department of Ophtalmology, Otorhinolaryngology, and Head and Neck Surgery - Faculty of Medicine of Ribeirão Preto - University of São \\ Paulo; Ribeirão preto, SP, Brazil; ${ }^{2}$ Department of Otorhinolaryngology - Head and Neck Surgery, University of Pennsylvania Health System; USA
}

\begin{abstract}
The recognition of sessile form of bacteria with particular features, known as biofilm, has given new insights to the understanding of pathogenesis of several chronic diseases, including Chronic Rhinosinusitis (CRS). In this article we review the main characteristics of biofilms, describe the current methods used to demonstrate biofilms in chronic rhinosinusitis and discuss the future directions of research in the field. Key-Words: Chronic rhinosinusitis, biofilm, planktonic bacteria.
\end{abstract}

For decades, Koch's four postulates were the paradigm to explain most of infectious diseases currently known. However, several recurrent and chronic infectious diseases could not be explained by the free-floating bacteria model of Koch's postulates. The discovery of a sessile conformation of bacteria known as biofilms in the 1980's has brought much interest to the study of the contribution of bacterial biofilms with many recurrent and chronic infectious diseases. A recent report by the Center for Disease Control and Prevention estimated that $65 \%$ of all infectious diseases in humans are related to biofilms [1]. Because of the increasing interest in biofilms over the last decades, we review the basic concepts related to biofilm microbiology and associated clinical implications and review the current evidence supporting the role of biofilms in chronic rhinosinusitis as well as future directions in research.

\section{What are Bacterial Biofilms?}

Bacteria can be found in nature in two distinct forms: freefloating cells denominated as planktonic or sessile forms known as biofilms. Biofilms are an adaptive phenotypic switch of prokaryotic life that virtually can be found in all living bacteria. Indeed, biofilms are the main mode of survival and bacterial proliferation. Morphologically, biofilms are characterized by a three dimensional complex of bacteria enclosed in a self-produced extracellular matrix formed by polysaccharides, nucleic acids, proteins and extracellular DNA (Figure 1). Approximately $90 \%$ of the biofilm mass is water with the remaining $10 \%$ of the mass being formed by extracellular matrix (8.5\%) and cells (1.5\%) [2].

The pores and water channels integral to the biofilm structure are the primary modes for nutrient and waste by product exchange with the external environment and are accomplished via both active and passive mechanisms. The physical structure of the biofilm establishes a decreasing oxygen and nutrient gradient from periphery to center which creates a Received on 21 February 2009; revised 7 May 2009.

Address for correspondence: Dr. Wilma T. Anselmo-Lima. Departament of Ophthalmology, Otorhinolaryngology, and Head and Neck Surgery. Faculty of Medicine of Ribeirão Preto, University of São Paulo. Avenida dos Bandeirantes, 3900, 14049-900, Ribeirão Preto-SP, Brazil. Telephone: +55(16) 3602-2862, Fax +55(16) 3602-2860. e-mail: wtalima@fmrp.usp.br.

The Brazilian Journal of Infectious Diseases 2009;13(3):232-235. (c) 2009 by The Brazilian Journal of Infectious Diseases and Contexto Publishing. All rights reserved. bacterial metabolic gradient in the same direction, with different susceptibility to antibiotics and to innate and adaptive host defenses [3]. These characteristics, allied to the ability of the biofilm community to detach viable bacteria and colonize distant niches, have given an increasing interest in biofilms in the understanding of persistent and recurrent infectious diseases.

\section{How are Biofilms Formed?}

Based on proteomic studies of Pseudomonas aeruginosa, five sequential and dynamic steps have been proposed to the formation of biofilms [3]. The first step starts with a random contact of bacteria with an inert or live surface. The initial contact is influenced by some features of the environment, such the presence of shearing forces or an air-liquid interface. Under certain environmental conditions, the bacteria use a signaling cascade to evoke phenotypic changes through the activation of selected genes involved in biofilm formation. This process is initiated with the production and secretion of signaling molecules that, depending on the concentration reached in the microenvironment, trigger the cascade necessary for biofilm formation. This tightly regulated process of cell-to-cell communication to initiate the biofilm phenotype is known as quorum-sensing.

Once the cascade is initiated, the second stage is the production of extracellular matrix that adheres the bacterial complex to the surface. The third stage is microcolony formation, the two dimensional clustering of bacteria. Once the microcolonies achieve a critical mass, vertical growth is initiated resulting in towers with intervening water channels and increase of polymeric extracellular matrix. Tower formation, however, is dependent of nutritional conditions of external environment that can either influence clonal cell growth or bacterial motility to form towers. In this step, colonizers like fungi and other bacteria can associate itself to the biofilm structure. In the last step, shearing forces as well as unknown conditions can induce detachment of free living bacteria to colonize far niches to maintain the bacterial cycle (Figure 2).

\section{What are the Advantages to Bacteria to Form a Biofilm?}

From an evolutional point of view, bacterial growth attached to a surface brings several advantages to survival and prokaryotic perpetuation. The attachment to a surface creates a certain degree of stability for survival, with a possibility to interact and cooperate as well as share genetic material with 
other cells within the biofilm community thereby greatly enhancing the gene pool. Another advantage is that bacteria within the biofilm are physically less susceptible to environmental factors, such as ultra-violet radiation, dehydration, variations of $\mathrm{pH}$ and osmolarity [3].

One of the most important features of biofilms is the high resistance to antibiotics and to host immune mechanisms such as less susceptibility to opsonization and phagocytosis $[4,5]$. In vitro tests have demonstrated that certain strains of bacteria in a biofilm state can be more than 1000 times more resistant to antibiotics compared to the minimum inhibitory concentrations levels of their planktonic counterparts [6]. One of the mechanisms of resistance is due to the physical barrier exerted by the polyssacharide matrix that blocks the diffusion of the compounds or inactivates the biocide activity of some agents. This is mainly important for reactive agents (i.e. superoxides) and immunoglobulins $[7,8]$. However, some studies have shown that many antibiotics are able to cross the exopolyssacaride matrix but do not have the expected antimicrobial activity [9]. This in part can be explained by the reduced metabolic activity found in the core of the biofilm resulting from lower concentrations of nutrients and oxygen. As most antibiotic act on active metabolic bacteria, like $\beta$ lactams, they would be expected not to have the highest effect in the core zones or "dormant" areas of the biofilm. This could explain the persistence of biofilm even after higher doses of appropriate antibiotic [10]. Other hypotheses to explain antibiotic resistance is that the water channels could pump the antibiotic out in an active manner [11].

Diseases that present with high resistance to conventional antibiotic therapy, with intermittent periods of acute exacerbations, difficult to identify and culture the microorganism, are the typical paradigm of biofilm-related diseases. Because of the long time adherence to a surface in addition to the ability to spread viable planktonic bacteria to far sites, biofilms can fit the pathophysiological model of several chronic and recurrent diseases. In this way, many studies have shown the relation of bacterial biofilms to dental plaques, urinary infections, prostatitis, pneumonia in cystic fibrosis patients, otitis media and, more recently, in chronic rhinosinusitis [12].

\section{Methods of Study and Evidence of Biofilms in Rhinosinusitis}

The understanding of biofilms has gained momentum as the methods of study have evolved. Currently, the main methods used to evaluate biofilm in rhinosinusitis are based on imaging studies (scanning electron microscopy, transmission electron microscopy, confocal scanning laser microscopy) and on methods that evaluate in vitro the formation and/or susceptibility of biofilms in 96-well plates assays [12], such as the Calgary Biofilm assay.

\section{Scanning Electron Microscopy (SEM) and Transmission Electron Microscopy (TEM)}

Much of the current knowledge about biofilms is due to the advances in imaging studies, especially the SEM. The ability to acquire images in high magnification, with structural microscopic details, is the main advantage of SEM. TEM, in turn, can elucidate ultra structural details of biofilms, like the composition and the interaction of extracellular matrix with the surface and cells in the vicinity.

However, both SEM and TEM have the disadvantage of artifacts inherent to the processing of the samples, which include dehydration and distortion of the surface. Another drawback is that SEM and TEM require very small pieces to be analyzed, which could add a sampling error bias.

The first study of biofilms in rhinosinusitis was published by Cryer et al. [13] in 2004, which identified bacterial biofilm in 4 of 16 patients with CRS by SEM. In another study from the same laboratory, Perloff and Palmer [14] evaluated the presence of biofilms in silastic stents removed from the frontal sinus recess after endoscopic sinus surgery (Figure 3). In this study, all 6 patients presented with biofilm on these sinus stents. Using TEM, Ferguson and Stolz [15] demonstrated the presence of bacterial biofilms in 2 of 4 patients with CRS. Also using SEM and TEM, Sanclement et al. [16] in 2005 demonstrated a prevalence of $80 \%(24 / 30)$ of biofilms in mucosal biopsies of CRS patients. None of the 4 control patients was found to be biofilm positive. Although potential artifacts can arise from the fixation and dehydration inherent to SEM processing, these authors claim that the conventional SEM processing does not change significantly the biofilm architecture and keep possible the recognition of biofilm on mucosal samples.

In children, Zuliani et al. [17] compared the presence of biofilms by SEM in adenoids in CRS versus Obstructive Sleep Apnea. CRS samples had $94.9 \%$ of the adenoid surface occupied by biofilm, in contrast to $1.9 \%$ in cases of obstructive sleep apnea. In this study they concluded that biofilms in the nasopharynx can be a reservoir for resistant bacteria and the elimination of this resource by adenoidectomy could explain the clinical improvement seen in these children.

\section{Confocal Scanning Laser Microscopy (CSLM)}

Another method that has gained interest in biofilm identification is the CSLM. Although the CSLM does not provide high imaging magnifications like SEM, its main advantage over the other methods is the possibility to visualize a specimen without fixation or dehydration of the sample, thereby minimizing possible architecture alterations of the biofilms. Another advantage is the possibility to stain bacterial cells or fungi selectively with fluorescent markers, such the probes used in in situ hybridization or nucleic acid markers to distinguish viable from non-viable cells.

Utilizing 4 different markers for fluorescent in situ hybridization (FISH), Sanderson et al. [18] demonstrated the presence of biofilms in 14 of 18 patients with CRS, including some samples with polymicrobial staining. However, 2 of 5 control samples showed positive staining for biofilms. Because the biofilms found in these control samples were significantly smaller than the CRS group, the authors suggest they might 
Figure 1. Scanning Electron Microphotograph. Pseudomonas aeruginosa (PA0-1) biofilm obtained after 24 hours of incubation over a polystyrene membrane. Note the bacilli (white arrow) enclosed in an extracellular matrix (black arrow) with water channels.

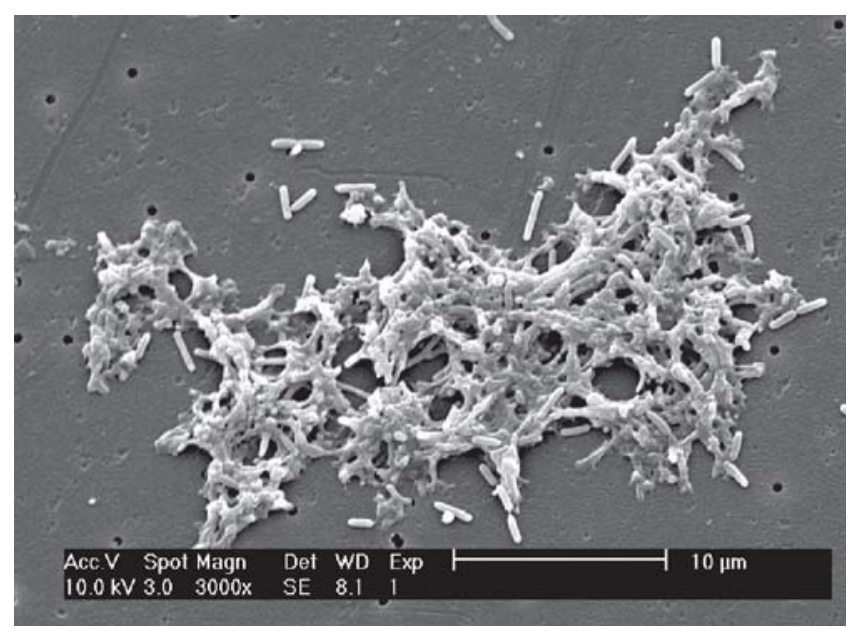

Figure 2. Biofilm formation cycle. (I) Planktonic bacteria; (II) Random contact of free-floating bacteria on a surface; (III) Formation of bacterial anchoring to the surface (IV); Growth of microcolonies; (V) Biofilm maturation and detachment of planktonic bacteria.



be only colonizers. Psaltis et al. [19], using CSLM associated with the Baclight LIVE/DEAD ${ }^{\circledR}$ kit staining, demonstrated that 17 of 38 patients (44.7\%) with CRS were biofilm positive and none of the 9 control patients showed evidence for biofilm.

\section{Modified Calgary Biofilm Assay}

The modified Calgary Biofilm Assay is a 96-well plate test evaluates the ability of bacteria to form biofilm on round pegs positioned over the plate. Originally describe to evaluate antibiotic
Figure 3. Scanning Electron Microphotograph. Silastic stent removed from the frontal sinus recess after the 7th post operative day, with biofilms on the surface.

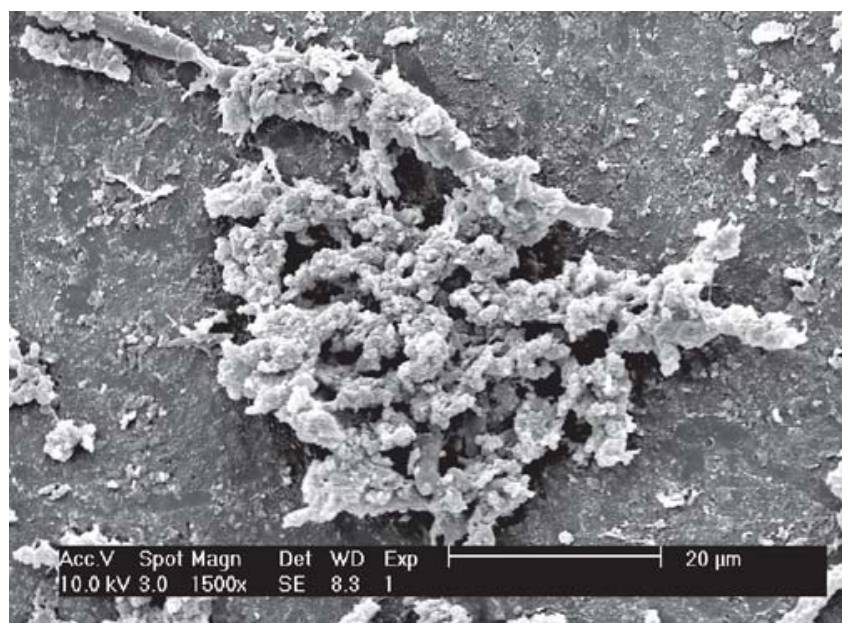

susceptibility and to compare the resistance to their planktonic counterparts [6], the modification described by Stepanovic et al. [20] consist in a semi-quantitative analysis of the amount of crystal violet absorbed by the biofilms grown on the pegs. The advantage of this method is the easy applicability and low cost. However, as an in vitro test, the biofilm formation evaluated on the pegs may not reflect the same behavior in vivo.

Besides the evidences in sinonasal mucosa, a study using the Calgary Biofilm Assay demonstrated a prevalence of $28.6 \%$ of bacterial biofilm formers in swabs collected from patients with CRS [21]. Using a similar method, Bendouah et al. [22] demonstrated that when Staphylococcus aureus or Pseudomonas aeruginosa recovered from CRS patients are able to form biofilm, they are associated with an unfavorable post operative evolution based on nasal endoscopy and on quality of life scores. In the same way, Psaltis et al. [23] demonstrated that the presence of biofilms on sinus mucosa of CRS patients were associated with worse symptoms and mucosal inflammation in the post-operative follow up.

\section{Future Perspectives in Research and Clinical Applications}

Although there is much evidence suggesting a link between biofilms and CRS in humans, there is little knowledge about its real contribution in the pathophysiology of CRS. There are no studies that clearly demonstrate which factors determine the persistence and growth of biofilm on the sinonasal mucosa of the host.

Due to the difficulty of studying biofilms on a viable tissue culture or even in animals few investigations have been able to successfully delve into evaluating the interactions between host and biofilms. Starner et al. [24] using broncho-epithelial human cells, demonstrated that $H$. influenza biofilms grown on 
these cell cultures evoked an inflammatory response with an increase of NF-kB, IL-8, TNF- $\alpha$, and MIP-3 $\alpha$ (macrophage inflammatory protein). Therefore, many studies are still required to understand the different mechanisms of interaction between host and biofilms, what drives the formation of biofilms in these patients, and development of an understanding of the humoral and cellular defense responses involved.

To better understand the behavior of biofilms in sinonasal mucosa, few studies using animal models have been published. Perloff and Palmer [25], demonstrated that maxillary sinusitis induced by $P$. aeruginosa in rabbits in different endpoints, until the $20^{\text {th }}$ day of infection, were associated with biofilm formation on sinus mucosa. Recently, Ha et al. [26] using a sheep model for sinusitis demonstrated that sinus ostium occlusion substantially promoted biofilm formation in the inoculated sinus.

Broadly, therapeutic intervention can be divided into: (1) possible manners to inhibit biofilm formation and (2) methods to eradicate biofilm already formed. Chiu et al. $[27,28]$ and Antunes et al. [29] have developed an animal biofilm sinusitis model with an indwelling irrigation catheter for screening antibiofilm activity of different topical agents, like antibiotics and/or surfactants. This model may develop into the workhorse for future topical drug development as it can be easily used to assess the efficacy and safety of anti-biofilm agents, like antiquorum sensing drugs [30].

Finally, the development of more feasible methods for biofilm detection could contribute with new perspectives and direction of therapies in patients that present with chronic and recurrent infectious diseases, such as CRS.

\section{Acknowledgements}

We thank the support from CAPES to E.T. (grant 4321/ 06-5). We thank Seana A. Wood and Andrew Scheller Jr. to the graphic illustrations (Philadelphia VA Medical Center, Medical Media).

\section{References}

1. Potera C. Forging a link between biofilm and disease. Science 1999; 283:1837-9.

2. Costerton J.W., Lewandowski Z., Caldwell D.E., et al. Microbial biofilms. Annual Rev Microbiol 1995; 49:711-45.

3. Hall-Stoodley L., Costerton J.W., Stoodley P. Bacterial biofilms: from the natural environment to infectious diseases. Nat Rev Microbiol 2004 Feb; 2(2):95-108.

4. Vuong C., Voyich J.M., Fischer E.R. et al. Polysaccharide intercellular adhesin (PIA) protects Staphylococcus epidermidis against major components of the human innate immune system. Cell Microbiol 2004 Mar; 6(3): 269-75.

5. Leid J.G., Shirtliff M.E., Costerton J.W., Stoodley P. Human leukocytes adhere, penetrate, and respond to Staphylococcus aureus biofilms. Infect Immun 2002;70:6339-45.

6. Ceri H., Olson M.E., Stremick C. et al. The Calgary Biofilm Device: New Technology for Rapid Determination of Antibiotic Susceptibilities of Bacterial Biofilms. J Clin Microbiol 1999;37(6): 1771-6.

7. Stewart P.S., Costerton J.W. Antibiotical resistance of bacteria in biofilms. Lancet 2001;358:135-8.

8. Costerton W., Veeh R., Shirtliff M. et al. The application of biofilm science to the study of and control of chronic bacterial infections. J Clin Invest 2003;112:1446-77.
9. Walters M.C., Roe F., Bugnicourt A. et al. Contributions of antibiotic penetration, oxygen limitation, and low metabolic activity to tolerance of Pseudomonas aeruginosa biofilm to ciprofloxacin and tobramycin. Antimicrob Agents Chemother 2003;47:317-23.

10. Spoering A.L., Lewis K. Biofilms and planktonic cells of Pseudomonas aeruginosa have similar resistance to killing by antimicrobials. J Bacteriol 2001;183(23):6746-51.

11. Gillis R.J., White K.G., Choi K.H. et al. Molecular basis of azithromyncin-resistent Pseudomonas aeruginosa biofilms. Antimicrob Agents Chemother 2005;49:3858-67.

12. Donlan R.M., Costerton J.W. Biofilms: survival mechanisms of clinically relevant microorganisms. Clin Microbiol Rev 2002;15(2):167-93.

13. Cryer J., Chipor I., Perloff J.R., Palmer J.N. Evidence of Bacterial Biofilms in Human Chronic Sinusitis. ORL J Otorhinolaryngol Relat Spec 2004;66(3):155-8.

14. Perloff J., Palmer J.N. Evidence of Bacterial Biofilms on Frontal Recess Stents in Patients with Chronic Rhinosinusitis. Am J Rhinol 2004 Nov-Dec;18(6):377-80.

15. Ferguson B.J., Stolz D.B. Demonstration of biofilm in human bacterial chronic rhinosinusitis. Am J Rhinol 2005 SepOct;19(5):452-7.

16. Sanclement J.A., Webster P., Thomas J., Ramadan H.H. Bacterial biofilms in surgical specimens of patients with chronic rhinosinusitis. Laryngoscope 2005 Apr; 115(4):578-82.

17. Zuliani G., Carron M., Gurrola J. et al. Identification of adenoid biofilms in chronic rhinosinusitis. Int J Pediatr Otorhinolaryngol 2006 Sep; 70(9):1613-7.

18. Sanderson A.R., Leid J.G., Hunsaker D. Bacterial biofilms on the sinus mucosa of human subjects with chronic rhinosinusitis. Laryngoscope 2006 Jul; 116(7):1121-6.

19. Psaltis A.J., Ha K.R., Beule A.G. et al. Confocal Scanning Laser Microscopy Evidence of Biofilms in Patients with Chronic Rhinosinusitis. Laryngoscope 2007 Jul; 117(7):1302-6.

20. Stepanovic S., Vukovic D., Dakic I. et al. A modified microtiterplate test for quantification of staphylococcal biofilm formation. J Microbiol Methods 2000 Apr; 40 (2):175-9

21. Prince A.A., Steiger J.D., Khalid A.N. et al. Prevalence of Biofilm forming bacteria in Chronic Rhinosinusitis. Am J Rhinol 2008, 22(3): 235-8.

22. Bendouah Z., Barbeau J., Hamad W.A., Desrosiers M. Biofilm formation by Staphylococcus aureus and Pseudomonas aeruginosa is associated with an unfavorable evolution after surgery for chronic sinusitis and nasal polyposis. Otolaryngol Head Neck Surg 2006 Jun; 134(6):991-6.

23. Psaltis A.J., Weitzel E.K., Ha K.R., Wormald P.J. The effect of bacterial biofilms on post-sinus surgical outcomes. Am J Rhinol 2008;22(1):1-6.

24. Starner T.D., Zhang N., Kim G. et al. Haemophilus influenzae Forms Biofilms on Airway Epithelia: Implications in Cystic Fibrosis. Am J Resp Crit Care Med 2006;174:213-20.

25. Perloff J., Palmer J.N. Evidence of bacterial biofilms in a rabbit model of sinusitis. Am J Rhinol 2005 Jan-Feb; 19(1):1-6.

26. Ha K.R., Psaltis A.J., Tan L., Wormald P.J. A sheep model for the study of biofilms in rhinosinusitis. Am J Rhinol 2007 May-Jun; 21(3):339-45. Erratum in: Am J Rhinol. 2007 Jul-Aug;21(4):519).

27. Chiu A.G., Antunes M.B., Feldman M., Cohen N.A. An animal model for the study of topical medications in sinusitis. Am J Rhinol 2007 Jan-Feb; 21(1):5-9.

28. Chiu A.G., Antunes M.B., Palmer J.N., Cohen N.A. Evaluation of the in vivo efficacy of topical tobramycin against Pseudomonas sinonasal biofilms. J Antimicrob Chemother 2007 Jun; 59(6):1130-4.

29. Antunes M.B., Feldman M.D., Cohen N.A., Chiu A.G. Dosedependent effects of topical tobramycin in an animal model of Pseudomonas sinusitis. Am J Rhinol 2007 Jul-Aug; 21(4):423-7.

30. Sperandio V. Novel approaches to bacterial infection therapy by interfering with bacteria-to-bacteria signaling. Expert Rev Anti Infect Ther 2007 Apr; 5(2):271-6. 